Проблеми математичного моделювання

та теорії диференціальних рівнянь

УДК 517.928: 533.6.013.2

\title{
АСИМПТОТИЧНО СУБОПТИМАЛЬНІ КЕРУВАННЯ СИСТЕМОЮ БЕНАРА В УЗАГАЛЬНЕНІЙ ЧАРУНЦІ КУЕТТА ПРИ ЇЇ ПЕРФОРАЦІЇ ТОНКИМИ ЦИЛІНДРАМИ
}

\author{
В. В. Гоцуленко
}

Iнститут підприємниитва "Стратегія", м. Жовті Води, 52201.

E-mail:gosul@ukr.net

Досліджується математична модель руху в'язкої рідини в узагальненій чарунці Куетта при перфорації її тонкими циліндрами з урахуванням граничного теплопідводу, та на їі основі побудова субоптимального керування для задачі граничного оптимального керування системою Бенара.

Ключові слова. Узагальнена чарунка Куетта, система Бенара, варіаційна збіжність, усереднення, асимптотично субоптимальне керування.

\section{1. Вступ}

Одним із найбільш відомих модельних об'єктів для дослідження руху нестискуваної рідини є чарунка Куетта [3]. Відомо, що до механізмів збудження руху рідини можна віднести як зміну напору (різницю тисків), так і вплив теплопідводу. Зазвичай математичною моделлю гравітаційної конвекції рідини в такій чарунці є система нелінійних диференціальних рівнянь типу Бенара. Проте чисельний та якісний аналіз розв'язків системи Бенара та пов'язаних із нею оптимізаційних задач досить ускладнений через ㄲï нелінійність і залишається актуальною проблемою. Проблеми чисельного моделювання таких процесів стають ще більш складними, якщо вихідна область має густо перфорований характер. Прототипом такої області може виступати узагальнена чарунка Куетта, яка є циліндричним тілом, перфорованим великою кількістю тонких циліндрів. Причому вважається, що кількість циліндрів тісно пов'язана з їх поперечними розмірами, а саме з ростом кількості внутрішніх циліндрів їх поперечні розміри прямують до нуля. Тому будь-яка дискретизація системи Бенара в такій області приводить до появи системи алгебраїчних рівнянь, розмірність якої не дозволяє коректно залучити чисельні процедури навіть на сучасних ЕОМ.

У зв'язку з цим є актуальною проблема якісного дослідження оптимізаційних задач для системи Бенара в таких густо перфорованих циліндричних областях (чарунці Куетта) та моделювання їх субоптимальних характеристик залежно від характеру перфорації.

\section{2. Визначення та класифікація узагальненої чарунки Куетта}

Позначимо через $\widetilde{\Omega}$ - обмежену відкриту множину в просторі $\mathbb{R}^{2}$ з достатньо гладкою границею $\partial \widetilde{\Omega}$. Нехай також $\widetilde{Y}=[-1 / 2,1 / 2)^{2}$, та $Q-$ компактна

(C) В. В. Гоцуленко, 2010 
підмножина в $\widetilde{Y}$ з гладкою границею $\partial Q$, причому $0 \in \operatorname{int} Q, A=B\left(\mathbf{0}, r_{0}\right)-$ відкрита куля з центром в нулі координат та радіусом $r_{0}<1 / 2$. При цьому також будемо вважати, що $Q \subset \subset A$. Нехай $\{\varepsilon\}-$ послідовність додатних чисел виду $\varepsilon=N^{-1}$, де $N \rightarrow \infty$. Введемо в розгляд такі множини:

$$
\left\{\begin{array}{l}
\Theta_{\varepsilon}=\left\{\mathbf{k}=\left(k_{1}, k_{2}\right) \in \mathbb{Z}^{2}: \varepsilon\left(r_{\varepsilon} Q+\mathbf{k}\right) \subset \subset \widetilde{\Omega}\right\} \\
\widetilde{\mathrm{T}}_{\varepsilon}^{\mathbf{k}}=\varepsilon\left(r_{\varepsilon} Q+\mathbf{k}\right), \mathbf{k} \in \Theta_{\varepsilon} \\
\widetilde{\mathrm{T}}_{\varepsilon}=\bigcup_{\mathbf{k} \in \Theta_{\varepsilon}} \widetilde{\mathrm{T}}_{\varepsilon}^{\mathbf{k}}, \mathrm{T}_{\varepsilon}=\widetilde{\mathrm{T}}_{\varepsilon} \times[0, \ell] \\
\widetilde{\Omega}_{\varepsilon}=\widetilde{\Omega} \backslash \widetilde{\mathrm{T}}_{\varepsilon}, \Omega_{\varepsilon}=\widetilde{\Omega}_{\varepsilon} \times(0, \ell) .
\end{array}\right.
$$

Ясно, що $\Omega_{\varepsilon}=\Omega \backslash\left[\bigcup_{\mathbf{k} \in \Theta_{\varepsilon}} \varepsilon\left(r_{\varepsilon} Q+\mathbf{k}\right) \times[0, \ell]\right]$, де $\Omega=\widetilde{\Omega} \times(0, \ell)$. Тут $\ell-$ висота чарунки Куетта, $r_{\varepsilon}$ - "поперечний розмір" тонких циліндрів. Відкрита множина $\Omega_{\varepsilon}$ в $\mathbb{R}^{3}$, яка періодично перфорована тонкими циліндрами $\mathrm{T}_{\varepsilon}^{\mathbf{k}}=$ $\widetilde{T}_{\varepsilon}^{\mathbf{k}} \times[0, \ell]$, називається узагальненою чарункою Куетта $[8,1]$. Оскільки кожен із циліндрів $\mathrm{T}_{\varepsilon}^{\mathbf{k}}$ утворено в результаті дії перетворення $\varepsilon$-гомотетії за першими двома координатами, $\mathrm{T}_{\varepsilon}^{\mathbf{k}}=\left\{\left(x_{1}, x_{2}, x_{3}\right):\left(x_{1}, x_{2}\right) \in \varepsilon\left(r_{\varepsilon} Q+\mathbf{k}\right), 0 \leq x_{3} \leq \ell\right\}$, то періодом перфорації множини $\Omega_{\varepsilon} \in$ чарунка $\Lambda=\varepsilon \widetilde{Y} \times[0, \ell]$. Позначимо через $\Gamma_{\varepsilon}$ - границю області $\Omega_{\varepsilon}$, тоді:

$$
\Gamma_{\varepsilon}=\Gamma_{\varepsilon}^{1} \bigcup \Gamma_{\varepsilon}^{2} \bigcup \Gamma^{3} \bigcup_{\mathbf{k} \in \Theta_{\varepsilon}} \widetilde{\partial} T_{\varepsilon}^{\mathbf{k}}
$$

де $\Gamma_{\varepsilon}^{1}=\widetilde{\Omega}_{\varepsilon} \times\{0\}, \Gamma_{\varepsilon}^{2}=\widetilde{\Omega}_{\varepsilon} \times\{\ell\}, \Gamma^{3}=\partial \widetilde{\Omega} \times[0, \ell], \widetilde{\partial} \mathrm{T}_{\varepsilon}^{\mathbf{k}}-$ бокова поверхня циліндра $\mathrm{T}_{\varepsilon}^{\mathrm{k}}$.

Структура усереднення як граничних задач, так і задач оптимального керування суттєво залежить від граничного значення $C_{0}=\lim _{\varepsilon \rightarrow 0} \sigma_{\varepsilon}$ параметра перфорації $\sigma_{\varepsilon}=\varepsilon^{2} \log \left(1 / r_{\varepsilon}\right)$ узагальненої чарунки Куетта $\Omega_{\varepsilon}$. Виділяються такі три типи перфорації узагальненої чарунки Куетта: $0<C_{0}<+\infty-$ критичний тип (перфорація циліндрами критичного розміру); $C_{0}=+\infty-$ перфорація тонкими циліндрами; $C_{0}=0$ - перфорація товстими циліндрами.

\section{3. Постановка задачі оптимального керування}

Нехай $\mathbf{z}_{\varepsilon}^{\partial}(x)$ і $b(x)$ задані функції, де малий параметр $\varepsilon>0$ набуває фіксованого значення. Об'єктом керування виступає нестаціонарний процес протікання в'язкої нестискуваної рідини в циліндрично перфорованій області $\Omega_{\varepsilon}$ 3 умовою прилипання на бічній поверхні циліндра. Позначимо через $\mathbf{y}_{\varepsilon}(x)-$

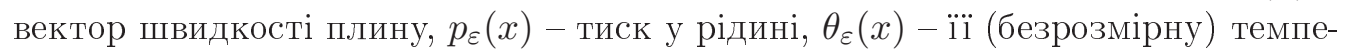
ратуру. Передбачається, що швидкість плину на вході та виході узагальненої чарунки Куетта $\Omega_{\varepsilon}$ є заданою, а процес протікання рідини, з урахуванням впливу температурних процесів, описується законом Бенара - Релея [7]. Задача керування полягає у знаходженні таких граничних значень швидкості $\vec{\alpha}_{\varepsilon}=\left(\alpha_{k_{1}}^{\varepsilon}, \alpha_{k_{2}}^{\varepsilon}, \ldots, \alpha_{k_{\varepsilon}}^{\varepsilon}\right)$ на бічних гранях тонких циліндрів $\mathrm{T}_{\varepsilon}^{\mathbf{k}}$ (за рахунок їх осьового обертання), при яких поле швидкостей в $\Omega_{\varepsilon}$ мало б задані властивості, зокрема, успадковувало б властивості, близькі до заданого розподілу 
$\mathbf{z}_{\varepsilon}^{\partial}(x)$. І рунтуючись на результатах роботи [7], приймемо наступну математичну модель руху рідини в області $\Omega_{\varepsilon}$ :

$$
\begin{gathered}
-\Delta \mathbf{y}_{\varepsilon}+\left(\mathbf{y}_{\varepsilon} \cdot \nabla\right) \mathbf{y}_{\varepsilon}=-\operatorname{grad} p_{\varepsilon}+\theta_{\varepsilon} \overrightarrow{\mathbf{e}_{3}}, \\
\operatorname{div} \mathbf{y}_{\varepsilon}=0, \\
-\Delta \theta_{\varepsilon}+\left(\mathbf{y}_{\varepsilon} \cdot \nabla\right) \theta_{\varepsilon}=\mathbf{y}_{\varepsilon} \cdot \overrightarrow{\mathbf{e}_{3}},
\end{gathered}
$$

з відповідними граничними умовами

$$
\begin{gathered}
\left.\mathbf{y}_{\varepsilon}\right|_{\Gamma_{\varepsilon}^{1}}=\mathbf{y}_{\varepsilon}^{1},\left.\quad \mathbf{y}_{\varepsilon}\right|_{\Gamma_{\varepsilon}^{2}}=\mathbf{y}_{\varepsilon}^{2},\left.\quad \mathbf{y}_{\varepsilon}\right|_{\Gamma^{3}}=0, \\
\left.\mathbf{y}_{\varepsilon}\right|_{\widetilde{\partial} \mathrm{T}_{\varepsilon}^{\mathbf{k}_{j}}}=\alpha_{\mathbf{k}_{j}}^{\varepsilon},\left.\quad \theta_{\varepsilon}\right|_{\widetilde{\partial} \mathrm{T}_{\varepsilon}^{\mathbf{k}_{j}}}=\beta_{\mathbf{k}_{j}}^{\varepsilon}(x)\left(j=\overline{1 ; J_{\varepsilon}}\right), \\
\left.\theta_{\varepsilon}\right|_{\Gamma_{\varepsilon}^{1}}=0,\left.\quad \theta_{\varepsilon}\right|_{\Gamma_{\varepsilon}^{2}}=0,\left.\quad \theta_{\varepsilon}\right|_{\Gamma^{3}}=\left.b\right|_{\Gamma^{3}} .
\end{gathered}
$$

Відносно функцій $\beta_{\mathbf{k}_{j}}^{\varepsilon}(x)$ припускається, що вектор $\vec{\beta}_{\varepsilon}=\left(\beta_{k_{1}}^{\varepsilon}, \beta_{k_{2}}^{\varepsilon}, \ldots, \beta_{k_{J_{\varepsilon}}}^{\varepsilon}\right) \epsilon$ звуженням деякої функції $b \in H^{1}(\Omega)$ на множину $\partial \Omega_{\varepsilon}$.

Для визначення слабого розв'язку крайової задачі (3.1)-(3.6) вводяться до розгляду такі форми:

$$
\begin{gathered}
a_{\varepsilon}(\mathbf{y}, \mathbf{v})=\int_{\Omega_{\varepsilon}} \nabla \mathbf{y}: \nabla \mathbf{v} d x \quad \forall \mathbf{y}, \mathbf{v} \in \mathbf{H}^{1}\left(\Omega_{\varepsilon}\right), \\
b_{\varepsilon}(\mathbf{y}, q)=-\int_{\Omega_{\varepsilon}} q \operatorname{div} \mathbf{y} d x \quad \forall \mathbf{y} \in \mathbf{H}^{1}\left(\Omega_{\varepsilon}\right), \quad \forall q \in L^{2}\left(\Omega_{\varepsilon}\right), \\
c_{\varepsilon}(\mathbf{y}, \mathbf{v}, \mathbf{w})=\int_{\Omega_{\varepsilon}}(\mathbf{y} \cdot \nabla) \mathbf{v} \cdot \mathbf{w} d x \quad \forall \mathbf{y}, \mathbf{v}, \mathbf{w} \in \mathbf{H}^{1}\left(\Omega_{\varepsilon}\right) .
\end{gathered}
$$

Означення 1. [5] Трійка функцій $\left(\mathbf{y}_{\varepsilon}, p_{\varepsilon}, \theta_{\varepsilon}\right) \in \mathbf{H}^{1}\left(\Omega_{\varepsilon}\right) \times L_{0}^{2}\left(\Omega_{\varepsilon}\right) \times H^{1}\left(\Omega_{\varepsilon}\right)$ називається слабим розв' язком граничної задачі (3.1)-(3.6), якщо виконуються граничні умови (3.4)-(3.6), та такі варіаційні рівності:

$$
\begin{aligned}
& a_{\varepsilon}\left(\mathbf{y}_{\varepsilon}, \mathbf{v}\right)+c_{\varepsilon}\left(\mathbf{y}_{\varepsilon}, \mathbf{y}_{\varepsilon}, \mathbf{v}\right)+b_{\varepsilon}\left(\mathbf{y}_{\varepsilon}, p_{\varepsilon}\right)=\int_{\Omega_{\varepsilon}} \theta_{\varepsilon}\left(\overrightarrow{\mathbf{e}_{3}} \cdot \mathbf{v}\right) d x \quad \forall \mathbf{v} \in \mathbf{H}_{0}^{1}\left(\Omega_{\varepsilon}\right), \\
& b_{\varepsilon}\left(\mathbf{y}_{\varepsilon}, q\right)=0 \quad \forall q \in L_{0}^{2}\left(\Omega_{\varepsilon}\right), \\
& \int_{\Omega_{\varepsilon}} \nabla \theta_{\varepsilon} \cdot \nabla \varphi d x+\int_{\Omega_{\varepsilon}}\left(\mathbf{y}_{\varepsilon} \cdot \nabla \theta_{\varepsilon}\right) \varphi d x=\int_{\Omega_{\varepsilon}}\left(\overrightarrow{\mathbf{e}_{3}} \cdot \mathbf{y}_{\varepsilon}\right) \varphi d x \quad \forall \varphi \in H_{0}^{1}\left(\Omega_{\varepsilon}\right) . \\
& \text { Функцію } \mathbf{u} \in \mathbf{H}_{\text {sol }}^{1}\left(\Omega_{\varepsilon}\right) \cap \mathbf{H}^{2}(\Omega) \text { таку, що }\left.\mathbf{u}\right|_{j=1} ^{\bigcup_{\varepsilon} \widetilde{\partial T}_{\varepsilon}^{\mathbf{k}_{j}}} \equiv \vec{\alpha}_{\varepsilon}, \text { будемо називати }
\end{aligned}
$$
прототипом для вектора керувань $\vec{\alpha}_{\varepsilon}$. Доведемо наступний результат.

Теорема 1. Нехай для вектора граничних умов $\vec{\alpha}_{\varepsilon}$ знайдетъся прототип $\mathbf{u} \in \mathbf{H}_{\text {sоl }}^{1}\left(\Omega_{\varepsilon}\right) \cap \mathbf{H}^{2}(\Omega)$, для якого виконуеться нерівність $\|\mathbf{u}\|_{\mathbf{H}^{2}(\Omega)} \leq \gamma$, при деякому $\gamma>0$, тоді знайдеться трійка

$$
\left(\mathbf{y}_{\varepsilon}, p_{\varepsilon}, \theta_{\varepsilon}\right) \in\left[\mathbf{H}_{\text {sol }}^{1}\left(\Omega_{\varepsilon}\right) \cap \mathbf{H}^{2}\left(\Omega_{\varepsilon}\right)\right] \times\left[H^{1}\left(\Omega_{\varepsilon}\right) \cap L_{0}^{2}\left(\Omega_{\varepsilon}\right)\right] \times H^{1}\left(\Omega_{\varepsilon}\right),
$$


maкa, щзо

$$
\begin{gathered}
\mathbf{y}_{\varepsilon}-\left.\mathbf{u}\right|_{\Omega_{\varepsilon}} \equiv \mathbf{y}_{\varepsilon}-\mathbf{u}_{\varepsilon} \in \mathbf{H}_{0, s o l}^{1}\left(\Omega_{\varepsilon}\right), \quad \theta_{\varepsilon}-\left.b\right|_{\Omega_{\varepsilon}} \equiv \theta_{\varepsilon}-b_{\varepsilon} \in H_{0}^{1}\left(\Omega_{\varepsilon}\right) \\
a_{\varepsilon}\left(\mathbf{y}_{\varepsilon}, v\right)+c_{\varepsilon}\left(\mathbf{y}_{\varepsilon}, \mathbf{y}_{\varepsilon}, \mathbf{v}\right)=\int_{\Omega_{\varepsilon}} \theta_{\varepsilon} \overrightarrow{\mathbf{e}_{3}} \cdot \mathbf{v} d x \quad \forall \mathbf{v} \in \mathbf{H}_{0, s o l}^{1}\left(\Omega_{\varepsilon}\right), \\
\nabla p_{\varepsilon}=\Delta \mathbf{y}_{\varepsilon}-\left(\mathbf{y}_{\varepsilon} \cdot \nabla\right) \mathbf{y}_{\varepsilon}+\theta_{\varepsilon} \overrightarrow{\mathbf{e}_{3}} \quad \text { нa } \mathcal{D}^{\prime}\left(\Omega_{\varepsilon}\right), \\
\int_{\Omega_{\varepsilon}} \nabla \theta_{\varepsilon} \cdot \nabla \varphi d x+\int_{\Omega_{\varepsilon}} \mathbf{y}_{\varepsilon} \cdot \nabla \theta_{\varepsilon} \varphi d x=\int_{\Omega_{\varepsilon}} \overrightarrow{\mathbf{e}_{\varepsilon}} \cdot \mathbf{y}_{\varepsilon} \varphi d x \quad \forall \varphi \in H_{0}^{1}\left(\Omega_{\varepsilon}\right) .
\end{gathered}
$$

Доведення. Існування розв'язку доведемо [5] методом Гальоркіна - спочатку будуємо деякий наближений розв'язок, а далі переходимо до границі. Зафіксуємо деякий прототип $\mathbf{u}$ та покладемо: $\mathbf{u}_{\varepsilon}=\left.\mathbf{u}\right|_{\Omega_{\varepsilon}}, \hat{\mathbf{y}}_{\varepsilon}=\mathbf{y}_{\varepsilon}-\mathbf{u}_{\varepsilon}$. Зауважимо, що, розглядаючи окремо останне рівняння із системи (3.3), де $\theta_{\varepsilon} \in$ невідома функція, а $\mathbf{y}_{\varepsilon}-$ фіксований елемент $з$ простору $\mathbf{H}_{\text {sol }}^{1}\left(\Omega_{\varepsilon}\right)$, для $\theta_{\varepsilon}$ [4] маємо подання $\theta_{\varepsilon}=b+\ell_{\varepsilon}\left(\hat{\mathbf{y}}_{\varepsilon}\right)$, де $\ell_{\varepsilon}: \mathbf{H}_{0}^{1}\left(\Omega_{\varepsilon}\right) \rightarrow H_{0}^{1}\left(\Omega_{\varepsilon}\right)$ - лінійний неперервний оператор.

Отже, для функції $\hat{\mathbf{y}}_{\varepsilon}$ маємо наступне рівняння:

$$
-\Delta \hat{\mathbf{y}}_{\varepsilon}+\left(\hat{\mathbf{y}}_{\varepsilon} \cdot \nabla\right) \hat{\mathbf{y}}_{\varepsilon}=-\nabla p_{\varepsilon}+\tilde{\ell}_{\varepsilon}\left(\hat{\mathbf{y}}_{\varepsilon}\right)+\mathbf{f}_{\varepsilon},
$$

де покладено:

$$
\tilde{\ell}_{\varepsilon}\left(\hat{\mathbf{y}}_{\varepsilon}\right)=\ell_{\varepsilon}\left(\hat{\mathbf{y}}_{\varepsilon}\right) \overrightarrow{\mathbf{e}_{3}}-\left(\mathbf{u}_{\varepsilon} \cdot \nabla\right) \hat{\mathbf{y}}_{\varepsilon}-\left(\hat{\mathbf{y}}_{\varepsilon} \cdot \nabla\right) \mathbf{u}_{\varepsilon}, \mathbf{f}_{\varepsilon}=\Delta \mathbf{u}_{\varepsilon}+b \overrightarrow{\mathbf{e}_{3}}
$$

Простір $\mathbf{H}_{0, \text { sol }}^{1}\left(\Omega_{\varepsilon}\right)$ є сепарабельним як замкнена підмножина простору $\mathbf{H}_{0}^{1}\left(\Omega_{\varepsilon}\right)$. Тому [5] існує лінійно незалежна послідовність функцій $\left\{\mathbf{w}_{\varepsilon, m}\right\}_{m \geq 1}$ з лінійного багатовиду $\left\{\mathbf{v} \in \mathbf{C}_{0}^{\infty}\left(\Omega_{\varepsilon}\right): \operatorname{div} \mathbf{v}(x)=\sum_{i=1}^{3} \partial \mathbf{v}_{i} / \partial x_{i}=0\right.$ на $\left.\Omega_{\varepsilon}\right\}$, яка тотальна в просторі $\mathbf{H}_{0, \text { sol }}^{1}\left(\Omega_{\varepsilon}\right)$.

Для кожного $m \geq 1$ визначимо наближений розв'язок $\hat{\mathbf{y}}_{\varepsilon, m}$ рівняння (3.7) за допомогою співвідношень $(k=\overline{1 ; m})$ :

$$
\hat{\mathbf{y}}_{\varepsilon, m}=\sum_{i=1}^{m} \xi_{i, m}^{\varepsilon} \mathbf{w}_{\varepsilon, m}, \quad \xi_{i, m}^{\varepsilon} \in \mathbf{R},
$$

$a_{\varepsilon}\left(\hat{\mathbf{y}}_{\varepsilon, m}, \mathbf{w}_{\varepsilon, k}\right)+c_{\varepsilon}\left(\hat{\mathbf{y}}_{\varepsilon, m}, \hat{\mathbf{y}}_{\varepsilon, m}, \mathbf{w}_{\varepsilon, k}\right)=\left\langle\tilde{\ell}_{\varepsilon}\left(\hat{\mathbf{y}}_{\varepsilon, m}\right), \mathbf{w}_{\varepsilon, k}\right\rangle+\left\langle\mathbf{f}_{\varepsilon}, \mathbf{w}_{\varepsilon, k}\right\rangle$.

Рівняння (3.8)-(3.9) являють собою систему нелінійних рівнянь відносно невідомих $\left\{\xi_{i, m}^{\varepsilon}\right\}_{i=\overline{1 ; m}}$. Існування розв'язку цієї системи випливає з результатів [5, с. 134].

Тепер, помножуючи (3.9) на $\xi_{i, m}^{\varepsilon}$, та складаючи одержані рівності для $k=1,2, \ldots, m$, будемо мати:

$$
\left\|\hat{\mathbf{y}}_{\varepsilon, m}\right\|_{\mathbf{H}_{0}^{1}\left(\Omega_{\varepsilon}\right)}^{2}+c_{\varepsilon}\left(\hat{\mathbf{y}}_{\varepsilon, m}, \hat{\mathbf{y}}_{\varepsilon, m}, \hat{\mathbf{y}}_{\varepsilon, m}\right)=\left\langle\tilde{\ell}_{\varepsilon}\left(\hat{\mathbf{y}}_{\varepsilon, m}\right), \hat{\mathbf{y}}_{\varepsilon, m}\right\rangle+\left\langle\mathbf{f}_{\varepsilon}, \hat{\mathbf{y}}_{\varepsilon, m}\right\rangle,
$$

звідки, враховуючи [5], що $c_{\varepsilon}\left(\hat{\mathbf{y}}_{\varepsilon, m}, \hat{\mathbf{y}}_{\varepsilon, m}, \hat{\mathbf{y}}_{\varepsilon, m}\right)=0$, одержується апріорна оцінка зверху:

$$
\left\|\hat{\mathbf{y}}_{\varepsilon, m}\right\|_{\mathbf{H}_{0}^{1}\left(\Omega_{\varepsilon}\right)} \leq\left\|\tilde{\ell}_{\varepsilon}\right\|_{\mathcal{L}\left(\mathbf{H}_{0}^{1}\left(\Omega_{\varepsilon}\right) ; H_{0}^{1}\left(\Omega_{\varepsilon}\right)\right)}+\left\|\mathbf{f}_{\varepsilon}\right\|_{\mathbf{L}^{2}\left(\Omega_{\varepsilon}\right)} .
$$


Використовуючи $[5,4]$ теорему Банаха - Алаоуглу, та теореми вкладення Соболєва, можливо, переходячи до підпослідовностей, одержати наступні співвідношення:

$$
\hat{\mathbf{y}}_{\varepsilon, m} \rightarrow \hat{\mathbf{y}}_{\varepsilon}^{*} \text { слабко в } \mathbf{H}_{0}^{1}\left(\Omega_{\varepsilon}\right) \text { та сильно в } \mathbf{L}^{2}\left(\Omega_{\varepsilon}\right) \text { при } m \rightarrow \infty .
$$

Відкіля випливають такі властивості:

$$
\begin{gathered}
c_{\varepsilon}\left(\hat{\mathbf{y}}_{\varepsilon, m}, \hat{\mathbf{y}}_{\varepsilon, m}, \mathbf{w}_{\varepsilon, k}\right) \rightarrow c_{\varepsilon}\left(\hat{\mathbf{y}}_{\varepsilon}^{*}, \hat{\mathbf{y}}_{\varepsilon}^{*}, \mathbf{w}_{\varepsilon, k}\right) \text { при } m \rightarrow \infty, \\
\left\langle\tilde{\ell}_{\varepsilon}\left(\hat{\mathbf{y}}_{\varepsilon, m}\right), \hat{\mathbf{y}}_{\varepsilon, m}\right\rangle \rightarrow\left\langle\tilde{\ell}_{\varepsilon}\left(\hat{\mathbf{y}}_{\varepsilon}^{*}\right), \hat{\mathbf{y}}_{\varepsilon}^{*}\right\rangle,\left\langle\mathbf{f}_{\varepsilon}, \hat{\mathbf{y}}_{\varepsilon, m}\right\rangle \rightarrow\left\langle\mathbf{f}_{\varepsilon}, \hat{\mathbf{y}}_{\varepsilon}^{*}\right\rangle \text { при } m \rightarrow \infty .
\end{gathered}
$$

3 (3.10)-(3.12) витікає, що для будь-якого $k=1,2, \ldots$

$$
a_{\varepsilon}\left(\hat{\mathbf{y}}_{\varepsilon}^{*}, \mathbf{w}_{\varepsilon, k}\right)+c_{\varepsilon}\left(\hat{\mathbf{y}}_{\varepsilon}^{*}, \hat{\mathbf{y}}_{\varepsilon}^{*}, \mathbf{w}_{\varepsilon, k}\right)=\left\langle\tilde{\ell}_{\varepsilon}\left(\hat{\mathbf{y}}_{\varepsilon}^{*}\right), \mathbf{w}_{\varepsilon, k}\right\rangle+\left\langle\mathbf{f}_{\varepsilon}, \hat{\mathbf{y}}_{\varepsilon}^{*}\right\rangle .
$$

Рівність (3.13) також виконується і для лінійних комбінацій функцій $\mathbf{w}_{\varepsilon, k}$.

Таким чином, (3.13) виконуеться при довільному $\mathbf{w}_{\varepsilon, k} \in \mathbf{H}_{0, s o l}^{1}\left(\Omega_{\varepsilon}\right)$. Отже $\hat{\mathbf{y}}_{\varepsilon}^{*}-$ розв'язок рівняння (3.7), а функції $\mathbf{y}_{\varepsilon}=\hat{\mathbf{y}}_{\varepsilon}^{*}+\mathbf{u}_{\varepsilon}$ та $\theta_{\varepsilon}=b_{\varepsilon}+\ell_{\varepsilon}\left(\hat{\mathbf{y}}_{\varepsilon}^{*}\right)-$ розв'язок вихідної задачі (3.1)-(3.6). Представлення для градієнта тиску

$$
\operatorname{grad} p_{\varepsilon}=\Delta \mathbf{y}_{\varepsilon}-\left(\mathbf{y}_{\varepsilon} \cdot \nabla\right) \mathbf{y}_{\varepsilon}+\theta_{\varepsilon} \overrightarrow{\mathbf{e}_{3}} \text { на } \mathcal{D}^{\prime}\left(\Omega_{\varepsilon}\right)
$$

$\epsilon$, як показано в [5], наслідком існування знайденого розв'язку $\hat{\mathbf{y}}_{\varepsilon}^{*}$.

Перейдемо тепер безпосередньо до розгляду задачі оптимізації. Нехай задано довільні функції $\mathbf{y}_{\varepsilon}^{*} \in \mathbf{H}_{\text {sol }}^{1}\left(\Omega_{\varepsilon}\right) \cap \mathbf{H}^{2}(\Omega)$, та $\mathbf{y}^{*} \in \mathbf{H}^{2}(\Omega)$, такі, що $\mathbf{y}_{\varepsilon}^{*} \rightarrow \mathbf{y}^{*}$ слабко в $\mathbf{H}^{2}(\Omega)$ при $\varepsilon \rightarrow 0$. Покладемо $\mathbf{y}_{\varepsilon}^{1}=\left.\mathbf{y}_{\varepsilon}^{*}\right|_{\Gamma_{\varepsilon}^{1}}$, та $\mathbf{y}_{\varepsilon}^{2}=\left.\mathbf{y}_{\varepsilon}^{*}\right|_{\Gamma_{\varepsilon}^{2}}$. Розглянемо наступну множину допустимих граничних керувань:

$$
\mathbb{U}_{\partial}^{\varepsilon}=\left\{\begin{array}{l|l}
\vec{\alpha}_{\varepsilon}=\left(\alpha_{\mathbf{k}_{1}}^{\varepsilon}, \ldots, \alpha_{\mathbf{k}_{J_{\varepsilon}}}^{\varepsilon}\right. & \begin{array}{l}
\alpha_{\mathbf{k}}^{\varepsilon}=\left.\mathbf{u}\right|_{\widetilde{\partial} \mathrm{T}_{\varepsilon}^{\mathbf{k}}} \forall \mathbf{k} \in \Theta_{\varepsilon}, \\
\forall \mathbf{u} \in \mathbf{H}_{s o l}^{1}\left(\Omega_{\varepsilon}\right) \cap \mathbf{H}^{2}(\Omega), \quad\|\mathbf{u}\|_{\mathbf{H}^{2}(\Omega)} \leq \gamma, \\
\left.\mathbf{u}\right|_{\Gamma_{\varepsilon}^{1}}=\mathbf{y}_{\varepsilon}^{1},\left.\mathbf{u}\right|_{\Gamma_{\varepsilon}^{2}}=\mathbf{y}_{\varepsilon}^{2},\left.\mathbf{u}\right|_{\Gamma^{3}}=0 .
\end{array}
\end{array}\right\},
$$

та введемо до розгляду наступний функціонал:

$$
\mathcal{I}_{\varepsilon}\left(\vec{\alpha}_{\varepsilon}, \mathbf{y}_{\varepsilon}, p_{\varepsilon}, \theta_{\varepsilon}\right)=\int_{\Omega_{\varepsilon}}\left|\mathbf{y}_{\varepsilon}-\mathbf{z}_{\varepsilon}^{\partial}\right|^{2} d x+\frac{\omega \varepsilon}{r_{\varepsilon}} \sum_{j=1}^{J_{\varepsilon}} \int_{\widetilde{\partial T}_{\varepsilon}^{\mathbf{k}_{j}}}\left|\alpha_{\mathbf{k}_{j}}^{\varepsilon}\right|^{2} d \mathcal{H}^{2} \rightarrow \text { inf },
$$

де $\omega>0$ - ваговий коефіцієнт, $\mathbf{z}_{\varepsilon}^{\partial}, \mathbf{z}^{\partial} \in \mathbf{L}^{2}(\Omega)$ - апріорно задані функції, причому $\mathbf{z}_{\varepsilon}^{\partial} \rightarrow \mathbf{z}^{\partial}$ в $\mathbf{L}^{2}(\Omega)$. Одержану задачу оптимального керування (3.14)(3.15) умовно позначимо символом $\left(\mathbb{P}_{\varepsilon}\right)$.

Означення 2. Четвірку $\left(\vec{\alpha}_{\varepsilon}, \mathbf{y}_{\varepsilon}, p_{\varepsilon}, \theta_{\varepsilon}\right)$ будемо називати припустимою (або припустимим розв'язком) задачі $\left(\mathbb{P}_{\varepsilon}\right)$, якщо $\vec{\alpha}_{\varepsilon} \in \mathbb{U}_{\partial}^{\varepsilon}, \vec{\beta}_{\varepsilon}=\left.b\right|_{\Omega_{\varepsilon}}, b \in H^{1}(\Omega)$, та трійка $\left(\mathbf{y}_{\varepsilon}, p_{\varepsilon}, \theta_{\varepsilon}\right) \in$ деяким слабким розв'язком граничної задачі (3.1)-(3.6). 
Задача граничного оптимального керування $\left(\mathbb{P}_{\varepsilon}\right)$ зводиться до аналізу еквівалентної задачі умовної мінімізації функціонала (3.15) на множині припустимих розв'язків:

$$
\Xi_{\varepsilon}=\left\{\begin{array}{c|c}
\left(\vec{\alpha}_{\varepsilon}, \mathbf{y}_{\varepsilon}, p_{\varepsilon}, \theta_{\varepsilon}\right) & \begin{array}{c}
\vec{\alpha}_{\varepsilon} \in \mathbb{U}_{\partial}^{\varepsilon}, \theta_{\varepsilon}-b \in H_{0}^{1}\left(\Omega_{\varepsilon}\right) \\
\mathbf{y}_{\varepsilon}-\mathbf{u}_{\varepsilon} \in \mathbf{H}_{0, \text { sol }}^{1}\left(\Omega_{\varepsilon}\right), \text { причому трійка } \\
\left(\mathbf{y}_{\varepsilon}, p_{\varepsilon}, \theta_{\varepsilon}\right) \in \mathbf{H}_{\text {sol }}^{1}\left(\Omega_{\varepsilon}\right) \times L_{0}^{2}\left(\Omega_{\varepsilon}\right) \times H^{1}\left(\Omega_{\varepsilon}\right)- \\
\in \text { слабим розв'язком задачі }(3.1)-(3.6)
\end{array}
\end{array}\right\},
$$

яку надалі умовно будемо позначати символом

$$
\left\langle\inf _{\left(\vec{\alpha}_{\varepsilon}, \mathbf{y}_{\varepsilon}, p_{\varepsilon}, \theta_{\varepsilon}\right) \in \Xi_{\varepsilon}} \mathcal{I}_{\varepsilon}\left(\vec{\alpha}_{\varepsilon}, \mathbf{y}_{\varepsilon}, p_{\varepsilon}, \theta_{\varepsilon}\right)\right\rangle
$$

Враховуючи [8] замкненість та обмеженість множини припустимих розв'язків (3.16), напівнеперервність знизу та рівномірну коерцитивність функціонала (3.15), як наслідок [4] маємо, що задача (3.17) є розв'язуваною тоді і тільки тоді, коли множина припустимих розв'язків $\Xi_{\varepsilon} \in$ не порожньою при будь-яких значеннях $\varepsilon>0$. Об'єднуючи цей факт із теоремою 1 , одержуємо твердження про існування припустимих розв'язків у задачі оптимального керування (3.14)-(3.15).

Теорема 2. Задача $\left(\mathbb{P}_{\varepsilon}\right)$ е розв'язуваною при будь-якому значенні $\varepsilon>0$.

\section{4. Формалізація задачі $\left(\mathbb{P}_{\varepsilon}\right)$ в термінах сингулярних мір}

Наша головна мета - побудова наближеного розв'язку задачі граничного оптимального керування $\left(\mathbb{P}_{\varepsilon}\right)$. Далі будуть визначені так звані асимптотично субоптимальні керування, однією з основних властивостей яких є поліпшення відповідних їм наближених розв'язків при $\varepsilon \rightarrow 0$. Ці керування будемо визначати через розв'язки усередненої (w- граничної) задачі.

Для ідентифікації граничного елемента послідовності припустимих розв'язків задач $\left(\mathbb{P}_{\varepsilon}\right)$ при $\varepsilon \rightarrow 0$ введемо до розгляду клас сингулярних періодичних мір Бореля $d \eta^{r}$ з чарункою періодичності $Y=[-1 / 2 ; 1 / 2)^{3}$, що сконцентровані на багатовиді $\partial Q^{r} \times[-1 / 2 ; 1 / 2)$, та для будь-якої гладкої функції $g(x)$ виконується рівність

$$
\int_{Y} g(x) d \eta^{r}(x)=r|\partial Q|_{H}^{-1} \int_{\partial Q^{r} \times[-1 / 2 ; 1 / 2)} g(x) d \mathcal{H}^{2},
$$

де $d \mathcal{H}^{2}$ - міра Хаусдорфа. Далі, розглядаючи міри $\eta_{\varepsilon}^{r_{\varepsilon}}(B)=\varepsilon^{3} \eta^{r_{\varepsilon}}\left(\varepsilon^{-1} B\right)$ для будь-якої борелевої множини $B \subset \mathbb{R}^{3}$, в [1] покажемо, що для довільного прототипу $\mathbf{u}_{\varepsilon}(x)$ вектора керувань $\vec{\alpha}_{\varepsilon}=\left(\alpha_{k_{1}}^{\varepsilon}, \alpha_{k_{2}}^{\varepsilon}, \ldots, \alpha_{k_{\varepsilon}}^{\varepsilon}\right)$ має місце тотожність

$$
\frac{\omega \varepsilon}{r_{\varepsilon}} \sum_{j=1}^{J_{\varepsilon}} \int_{\widetilde{\partial} T_{\varepsilon}^{\mathrm{k}_{j}}}\left|\alpha_{\mathrm{k}_{j}}^{\varepsilon}\right|^{2} d \mathcal{H}^{2}=\omega|\partial Q|_{H} \int_{\Omega}\left|\mathbf{u}_{\varepsilon}\right|^{2} d \eta_{\varepsilon}^{r_{\varepsilon}}
$$


де $|\partial Q|_{H}$ - міра Хаусдорфа багатовиду $\partial Q$. Таким чином, функціонал (3.15) можна подати в еквівалентній формі:

$$
\mathcal{I}_{\varepsilon}\left(\vec{\alpha}_{\varepsilon}, \mathbf{y}_{\varepsilon}, p_{\varepsilon}, \theta_{\varepsilon}\right)=\int_{\Omega_{\varepsilon}}\left|\mathbf{y}_{\varepsilon}-\mathbf{z}_{\varepsilon}^{\partial}\right|^{2} d x+\omega|\partial Q|_{H} \int_{\Omega}\left|\mathbf{u}_{\varepsilon}\right|^{2} d \eta_{\varepsilon}^{r_{\varepsilon}} .
$$

Означення 3. [8, 1]. Послідовність допустимих граничних керувань

$$
\left\{\vec{\alpha}_{\varepsilon} \in \mathbf{U}_{\varepsilon}\right\}_{\varepsilon>0}
$$

$\mathrm{w}_{a}$ - збігається до деякої функції $\mathbf{a}_{0}$, якщо знайдеться ㄲï послідовність прототипів $\left\{\mathbf{u}_{\varepsilon} \in \mathbf{H}^{2}(\Omega) \cap \mathbf{L}^{2}\left(\Omega, d \eta_{\varepsilon}^{r(\varepsilon)}\right)\right\}$, яка збігається до а $\mathbf{a}_{0}$ слабко в $\mathbf{H}^{2}(\Omega)$.

Означення 4. $[8,1]$ Послідовність допустимих граничних керувань

$$
\left\{\vec{\alpha}_{\varepsilon} \in \mathbf{U}_{\varepsilon}\right\}_{\varepsilon>0}
$$

$\mathrm{w}_{b}$ - збігається до деякої функції $\mathbf{b}_{0} \in \mathbf{L}^{2}(\Omega)$, якщо знайдеться її послідовність прототипів $\left\{\mathbf{u}_{\varepsilon} \in \mathbf{H}^{2}(\Omega) \cap \mathbf{L}^{2}\left(\Omega, d \eta_{\varepsilon}^{r(\varepsilon)}\right)\right\}_{\varepsilon>0}$, що збігається до $\mathbf{b}_{0}$ слабко в $\mathbf{L}^{2}\left(\Omega, d \eta_{\varepsilon}^{r}\right)$.

Зв'язок та коректність наведених вище $\mathrm{w}_{a} \mathrm{i} \mathrm{w}_{b}$ збіжностей гарантується таким твердженням [1]: будь-яка послідовність припустимих граничних керувань $\left\{\vec{\alpha}_{\varepsilon} \in \mathbf{U}_{\varepsilon}\right\}_{\varepsilon>0}$ містить підпослідовність, для якої їі $\mathrm{w}_{a}$ i $\mathrm{w}_{b}$ границі $\epsilon$ рівними майже скрізь у $\Omega$, причому довільна така послідовність є передкомпактною відносно $\mathrm{w}_{a}$ збіжності.

Означення 5. $[8,1]$. Послідовність припустимих розв'язків

$$
\left\{\left(\vec{\alpha}_{\varepsilon}, \mathbf{y}_{\varepsilon}, p_{\varepsilon}, \theta_{\varepsilon}\right) \in \Xi_{\varepsilon}\right\}_{\varepsilon>0}
$$

w- збігається до четвірки $(\mathbf{u}, \mathbf{y}, p, \theta) \in \mathbf{H}^{2}(\Omega) \times \mathbf{H}^{1}(\Omega) \times L_{0}^{2}(\Omega) \times H^{1}(\Omega)$ при $\varepsilon \rightarrow 0$ (в символьному записі $\mathbf{w}-\operatorname{Lim}_{\varepsilon \rightarrow 0}\left(\vec{\alpha}_{\varepsilon}, \mathbf{y}_{\varepsilon}, p_{\varepsilon}, \theta_{\varepsilon}\right)=(\mathbf{u}, \mathbf{y}, p, \theta)$ ), якщо знайдеться відповідна послідовність прототипів $\left\{\left(\mathbf{u}_{\varepsilon}, \breve{\mathbf{y}}_{\varepsilon}, \breve{p}_{\varepsilon}, \breve{\theta}_{\varepsilon}\right) \in \widehat{\Xi}_{\varepsilon}\right\}_{\varepsilon>0}$, яка збігається до четвірки функцій $(\mathbf{u}, \mathbf{y}, p, \theta)$ в такому сенсі : (i) $\mathbf{u}_{\varepsilon} \stackrel{\mathrm{w}_{a}}{\longrightarrow} \mathbf{u}$ в $\mathbf{H}^{2}(\Omega)$; (ii) $\breve{p}_{\varepsilon} \rightarrow p$ в $L_{0}^{2}(\Omega)$; (iii) $\breve{\mathbf{y}}_{\varepsilon} \rightarrow \mathbf{y}$ в $\mathbf{H}^{1}(\Omega)$; (iiii) $\breve{\theta}_{\varepsilon} \rightarrow \theta$ в $H^{1}(\Omega)$.

Тут символом " " позначено дію відповідного оператора продовження в множину $\Omega$, існування якого є наслідком сильної зв'язності [2] множини $\Omega_{\varepsilon}$. Відносно w-збіжності при довільній перфорації множини $\Omega_{\varepsilon}$ має місце результат [1].

Теорема 3. Нехай $\left\{\left(\vec{\alpha}_{\varepsilon}, \mathbf{y}_{\varepsilon}, p_{\varepsilon}, \theta_{\varepsilon}\right) \in \Xi_{\varepsilon}\right\}_{\varepsilon>0}-$ послідовність припустимих розв'язків для задач $\left\{\left(\mathbb{P}_{\varepsilon}\right)\right\}_{\varepsilon>0}$. Тоді існують підпослідовність

$$
\left\{\left(\vec{\alpha}_{\varepsilon^{\prime}}, \mathbf{y}_{\varepsilon^{\prime}}, p_{\varepsilon^{\prime}}, \theta_{\varepsilon^{\prime}}\right)\right\}_{\varepsilon^{\prime}>0}
$$

та четвірка функиій $(\mathbf{u}, \mathbf{y}, p, \theta) \in \mathbf{H}^{2}(\Omega) \times \mathbf{H}^{1}(\Omega) \times L_{0}^{2}(\Omega) \times H^{1}(\Omega)$ такі, що справедлива рівність $\mathbf{w}-\operatorname{Lim}_{\varepsilon \rightarrow 0}\left(\vec{\alpha}_{\varepsilon}, \mathbf{y}_{\varepsilon}, p_{\varepsilon}, \theta_{\varepsilon}\right)=(\mathbf{u}, \mathbf{y}, p, \theta)$, та мае місие включення $\mathbf{y}-\mathbf{u} \in \mathbf{H}_{0, \text { sol }}^{1}(\Omega)$. 


\section{5. Асимптотично субоптимальні керування в узагальненій чарунці Куетта}

Головне питання, яке ми розглядаємо в цьому розділі, торкається вивчення наближень до оптимальних керувань початкової задачі граничного оптимального керування $\left(\mathbb{P}_{\varepsilon}\right)$ для будь-якого малого параметра $\varepsilon>0$. Зосередимо нашу увагу на вивченні субоптимальних керувань, які з апріорно заданою точністю мінімізують початковий функціонал $\mathcal{I}_{\varepsilon}\left(\mathbf{u}_{\varepsilon}^{s u b}, \mathbf{y}_{\varepsilon}^{s u b}, p_{\varepsilon}^{\text {sub }}, \theta_{\varepsilon}^{s u b}\right)$.

Для цього введемо таке визначення:

Означення 6. Будемо говорити, що функція $\vec{\alpha}_{\varepsilon}^{s u b}=\left(\alpha_{\mathbf{k}_{1}}^{s u b}, \alpha_{\mathbf{k}_{2}}^{s u b}, \ldots, \alpha_{\mathbf{k}_{J_{\varepsilon}}}^{s u b}\right) \in$ асимптотитично субоптимальним керуванням для задачі $\left(\mathbb{P}_{\varepsilon}\right)$, якщо

$$
\alpha_{\mathbf{k}_{j}}^{s u b} \in \mathbf{H}^{1 / 2}\left(\widetilde{\partial} \mathrm{T}_{\varepsilon}^{\mathbf{k}_{j}}\right), \quad \int_{\widetilde{\partial} \mathrm{T}_{\varepsilon}^{\mathbf{k}_{j}}} \mathbf{n} \cdot \alpha_{\mathbf{k}_{j}}^{s u b} d \mathcal{H}^{2}=0, \quad \forall=1, \ldots \mathrm{J}_{\varepsilon}
$$

і для будь-якого $\delta>0$ знайдеться $\varepsilon_{0}>0$ таке, що

$$
\left|\inf _{\left(\vec{\alpha}_{\varepsilon}, \mathbf{y}_{\varepsilon} p_{\varepsilon}, \theta_{\varepsilon}\right) \in \Xi_{\varepsilon}} \mathcal{I}_{\varepsilon}\left(\vec{\alpha}_{\varepsilon}, \mathbf{y}_{\varepsilon}, p_{\varepsilon}, \theta_{\varepsilon}\right)-\mathcal{I}_{\varepsilon}\left(\vec{\alpha}_{\varepsilon}^{s u b}, \mathbf{y}_{\varepsilon}^{s u b}, p_{\varepsilon}^{s u b}, \theta_{\varepsilon}^{s u b}\right)\right|<\delta \quad \forall \varepsilon<\varepsilon_{0},
$$

де $\mathbf{y}_{\varepsilon}^{s u b}=\mathbf{y}_{\varepsilon}\left(\vec{\alpha}_{\varepsilon}^{s u b}\right), p_{\varepsilon}^{s u b}=p_{\varepsilon}\left(\vec{\alpha}_{\varepsilon}^{s u b}\right), \theta_{\varepsilon}^{s u b}=\theta_{\varepsilon}\left(\vec{\alpha}_{\varepsilon}^{s u b}\right)-$ розв'язок вихідної задачі (3.14)-(3.15), що відповідає допустимому керуванню $\vec{\alpha}_{\varepsilon}=\vec{\alpha}_{\varepsilon}^{s u b}$.

Для побудови субоптимальних керувань будемо використовувати підхід, заснований на варіаційній w-збіжності задач умовної мінімізації. Беручи це до уваги, вивчимо асимптотичну поведінку задачі $\left(\mathbb{P}_{\varepsilon}\right)$ коли $\varepsilon \rightarrow 0$. Подамо задачу $\left(\mathbb{P}_{\varepsilon}\right)$ при різних значеннях параметра $\varepsilon$ у формі наступної послідовності задач умовної мінімізації:

$$
\left\{\left\langle\inf _{\left(\vec{\alpha}_{\varepsilon}, \mathbf{y}_{\varepsilon}, p_{\varepsilon}, \theta_{\varepsilon}\right) \in \Xi_{\varepsilon}} \mathcal{I}_{\varepsilon}\left(\vec{\alpha}_{\varepsilon}, \mathbf{y}_{\varepsilon}, p_{\varepsilon}, \theta_{\varepsilon}\right)\right\rangle\right\}_{\varepsilon>0} .
$$

Основні властивості варіаційної w-граничної задачі

$$
\left\langle\inf _{(\mathbf{u}, \mathbf{y}, p, \theta) \in \Xi_{0}} \mathcal{I}_{0}(\mathbf{u}, \mathbf{y}, p, \theta)\right\rangle
$$

для послідовності (5.3) наведені в наступній теоремі (див., $[9,8,10,11]$ ).

Теорема 4. Нехай задача (5.4) е варіаційною $\mathrm{w}$-границею послідовності (5.3). Нехай також $\left\{\left(\bar{\alpha}_{\varepsilon}^{0}, \mathbf{y}_{\varepsilon}^{0}, p_{\varepsilon}^{0}, \theta_{\varepsilon}^{0}\right) \in \Xi_{\varepsilon}\right\}_{\varepsilon>0}-$ послідовність оптимальних розв'язків задач $\left(\mathbb{P}_{\varepsilon}\right)$. Тоді існуе набір $\left(\mathbf{u}^{0}, \mathbf{y}^{0}, p^{0}, \theta^{0}\right) \in \Xi_{0}$ такий, що

$$
\begin{gathered}
\mathrm{w}-\operatorname{Lim}_{\varepsilon \rightarrow 0}\left(\vec{\alpha}_{\varepsilon}, \mathbf{y}_{\varepsilon}, p_{\varepsilon}, \theta_{\varepsilon}\right)=(\mathbf{u}, \mathbf{y}, p, \theta), \\
\inf _{(\mathbf{u}, \mathbf{y}, p, \theta) \in \Xi_{0}} \mathcal{I}_{0}(\mathbf{u}, \mathbf{y}, p, \theta)=\mathcal{I}_{0}\left(\mathbf{u}^{0}, \mathbf{y}^{0}, p^{0}, \theta^{0}\right)= \\
=\lim _{\varepsilon \rightarrow 0} \inf _{\left(\bar{\alpha}_{\varepsilon}, \mathbf{y}_{\varepsilon}, p_{\varepsilon}, \theta_{\varepsilon}\right) \in \Xi_{\varepsilon}} \mathcal{I}_{\varepsilon}\left(\vec{\alpha}_{\varepsilon}, \mathbf{y}_{\varepsilon}, p_{\varepsilon}, \theta_{\varepsilon}\right),
\end{gathered}
$$


де $\mathrm{K}-\operatorname{Lim}_{\varepsilon \rightarrow 0} \Xi_{\varepsilon}=\Xi_{0}$ - границя в сенсі Куратовсъкого [6] послідовності множин $\left\{\Xi_{\varepsilon}\right\}_{\varepsilon>0}, \mathcal{I}_{0}: \Xi_{0} \rightarrow \overline{\mathbb{R}}$ - варіаційна S-границя послідовності функціоналів (4.1), більше того [1],

$$
\mathcal{I}_{0}(\mathbf{u}, \mathbf{y}, p, \theta)=\int_{\Omega}\left|\mathbf{y}-\mathbf{z}_{\partial}\right|^{2} d x+\omega|\partial Q|_{H} \int_{\Omega}|\mathbf{u}|^{2} d x,
$$

за умови, що $\mathbf{z}_{\varepsilon}^{\partial} \rightarrow \mathbf{z}_{\partial}$ сильно в $\mathbf{L}^{2}(\Omega)$.

Структура субоптимальних керувань, визначених в означенні 6 , одержана в наступній теоремі [1].

Теорема 5. Нехай четвірка $\left(\mathbf{u}^{0}, \mathbf{y}^{0}, p^{0}, \theta_{0}\right) \in \Xi_{0}$ е оптимальним розв'язком w-yсередненої задачі (5.4). Тоді існуе лінійний неперервний оператор $\Lambda_{\varepsilon}$ : $\mathbf{H}_{\text {sol }}^{1}(\Omega) \mapsto \mathbf{H}_{\text {sol }}^{1}\left(\Omega_{\varepsilon}\right)$, такий, що функція

$$
\vec{\alpha}_{\varepsilon}^{s u b}=\left(\alpha_{\mathbf{k}_{1}}^{s u b}, \alpha_{\mathbf{k}_{2}}^{s u b}, \ldots, \alpha_{\mathbf{k}_{\varepsilon}}^{s u b}\right)=\left.\Lambda_{\varepsilon}\left(\mathbf{u}^{0}\right)\right|_{\partial \Omega_{\varepsilon}}
$$

е асимптотично субоптимальним керуванням для початкової задачі $\left(\mathbb{P}_{\varepsilon}\right)$ в сенсі означення 6.

Зауваження 1. В праці [1] для критичного випадку $0<C_{0}<\infty$ перфорації узагальненої чарунки Куетта $\Omega_{\varepsilon}$ було доведено, що w-гранична задача умовної мінімізації (5.4) до послідовності задач граничного оптимального керування $\left(\mathbb{P}_{\varepsilon}\right)$ може бути представлена також у вигляді задачі оптимального, але розподіленого керування Діріхле $\mathbf{u} \in \mathbf{U}=\left\{\mathbf{u} \in \mathbf{H}^{2}(\Omega):\|\mathbf{u}\|_{\mathbf{H}^{2}(\Omega)} \leq \gamma\right\}$ по всій області $\Omega$ системою Біркмана, яка описується крайовою задачею

$$
\begin{gathered}
-\Delta \mathbf{y}+\frac{2 \pi}{C_{0}}(\mathbf{y}-\mathbf{u})+(\mathbf{y} \cdot \nabla) \mathbf{y}+\nabla p=\theta \overrightarrow{\mathbf{e}_{3}} \text { в } \Omega ; \\
\operatorname{div} \mathbf{y}=0 \quad \text { в } \Omega ; \\
\left.\mathbf{y}\right|_{\partial \Omega}=\left.\mathbf{u}\right|_{\partial \Omega} ; \\
-\Delta \theta+\frac{2 \pi}{C_{0}}(\theta-b)+\mathbf{y} \cdot \nabla \theta=\mathbf{y} \cdot \overrightarrow{\mathbf{e}_{3}} ; \\
\left.\theta\right|_{\partial \Omega}=\left.b\right|_{\partial \Omega} .
\end{gathered}
$$

та цільовим функціоналом (5.7).

Таким чином, переходячи до границі при $C_{0} \rightarrow+\infty$ у варіаційній постановці рівнянь (5.9) і (5.12) та враховуючи структуру граничного функціонала (5.7), у випадку перфорації узагальненої чарунки Куетта тонкими ціліндрами, маємо такий результат:

Теорема 6. Нехай $C_{0}=+\infty$, тоді задача (5.4), яка е варіаційною w-границеюо послідовності задач умовної мінімізачій (5.3), що відповідають задачам оптимального керування $\left\{\left(\mathbb{P}_{\varepsilon}\right)\right\}_{\varepsilon>0}$, може бути подана як наступна ізопериметрична задача варіачійного числення:

мінімізувати інтеграл

$$
\int_{\Omega}|\mathbf{u}(x)|^{2} d x \rightarrow \inf ,
$$


при обмеженнях

$$
\left\{\begin{array}{c|c}
\mathbf{u} \in \mathbf{H}^{2}(\Omega) \mid \begin{array}{c}
\|\mathbf{u}\|_{\mathbf{H}^{2}(\Omega)} \leq \gamma, \nabla \cdot \mathbf{u}=0 \text { на } \Omega, \\
\left.\mathbf{u}\right|_{\Gamma^{1}}=\left.\mathbf{y}^{*}\right|_{\Gamma^{1}},\left.\mathbf{u}\right|_{\Gamma^{2}}=\left.\mathbf{y}^{*}\right|_{\Gamma^{2}},\left.\mathbf{u}\right|_{\Gamma^{3}}=0, \\
\mathbf{y}^{*} \cdot \mathbf{n}=0 \text { на } \Gamma^{1} \cup \Gamma^{2}
\end{array}
\end{array}\right\} .
$$

\section{6. Висновки}

Встановлено, що у випадку перфорації узагальненої чарунки Куетта $\Omega_{\varepsilon}$ тонкими циліндрами (тобто при $C_{0}=+\infty$ ), w-граничною задачею (за допомогою якої будуються пропоновані асимптотично субоптимальні керування) для задачі оптимального керування (3.14)-(3.15) є ізопериметрична задача (5.14)-(5.15) варіаційного числення, яка розглядається в однорідній області $\Omega$.

\section{Бібліографічні посилання}

1. Гочуленко В. В. Субоптимальное граничное управление системой Бенара в цилиндрически перфорированной области /В. В. Гоцуленко, П. И. Когут // Укр. мат. вест. - 2009. - Т. 6, № 4. - С. 436-474.

2. Марченко В. А., Хруслов Е. Я. Краевые задачи в областях с мелкозернистой границей. - К. : Наукова думка, 1974.

3. Рихтмайер Р. Принципы современной математической физики /Р. Рихтмайер. - М. : Мир, т. 2, 1984. - 375 с.

4. Фурсиков A. В. Оптимальное управление распределенными системами. Теория и приложения /А. В. Фурсиков.- Новосибирск : Научная книга, 1999. -560 с.

5. Темам Р. Уравнения Навье-Стокса / Р. Темам.- М. : Мир, 1981. - 435 с.

6. Attouch H.Variational Convergence for Functional and Operators /H. Attouch // Appl. Math. Ser., Pitman. Boston - London, 1984.

7. Birnir B., Svanstedt N. Existence theory and strong attractors for the RayleighBénard problem with a large aspect ratio // Discrete and Continuous Dynamical Systems, 2004, Vol.10, No.1-2.- P. 55-74.

8. D'Apice C., De Maio U., Kogut P.I. Boundary velocity suboptimal control of incompressible flow in cylindrically perforated domain // Discrete and Continuous Dynamical Systems, Series B, Vol. 11, No. 2, 2009, 283-314.

9. D'Apice C., De Maio U., Kogut P.I. Suboptimal boundary controls for elliptic equation in critically perforated domain // Annales de l'institut Henri Poincaré :(C) Analyse non Linéare, Vol.25, Issue 6, 2008, 1073-1101.

10. Kogut P.I., Leugering G. On the homogenization of optimal control problems on periodic graphs// Lecture Notes in Pure and Applied Mathematics, 2007, Vol. 252, P. 55-74.

11. Kogut P.I., Leugering G. Asymptotic Analysis of State Constrained Semilinear Optimal Control Problems// Journal of Optimization Theory and Applications (JOTA), 2007, Vol. 135, No. 2, P. 301-321. 